\title{
Reliability and validity of rasterstereography measurement for spinal alignment in healthy subjects
}

\author{
Yoon-Sil Yi ${ }^{a}$, Seul-Ki Yoo ${ }^{b}$, Da-Gam Lee ${ }^{b}$, Dae-Sung Park ${ }^{b}$ \\ ${ }^{a}$ Department of Physical Therapy, The Graduate School, Konyang University, Daejeon, Republic of Korea \\ ${ }^{b}$ Department of Physical Therapy, Konyang University, Daejeon, Republic of Korea
}

Objective: The Back Mapper is one type of Rasterstereography and it can be used in the clinic without radiation exposure. The purpose of our study was to prove the reliability and validity of the Back Mapper and to compare it with the Spinal Mouse, which is an assessment tool for spinal curvatures using a wheeled mouse, and the Cobb angle by X-ray.

Design: Cross-sectional study.

Methods: Twenty healthy adults participated in the test to investigate for the inter-rater reliability, intra-rater reliability, and concurrent validity. The tests were performed with assessment devices for scoliosis such as the Back Mapper, Spinal Mouse and Cobb's angle. Data was analyzed by an intraclass correlation coefficient (ICC) value and a standard error of measurement for reliability and correlation analysis for validity.

Results: Intra-rater reliability of the Back Mapper was good (Cronbach's $\alpha=0.821-0.984$, ICC $=0.696-0.969)$ except for assessing the lordotic angle. Inter-rater reliability was good (Cronbach's $\alpha=0.870-0.958, \mathrm{ICC}=0.770-0.919$ ) in assessment for trunk imbalance, rotation of scapulae, thoracic angle, lumbar angle, and kyphotic angle. The kyphotic angle in the Spinal Mouse had a significant correlation icompared with the Back Mapper $(\mathrm{r}=0.510, p<0.05)$, and the Cobb's angle from an X-ray had a significant correlation with trunk inclination $(\mathrm{r}=0.532, p<0.05)$.

Conclusions: These findings provide good intra-reliability of the Back Mapper in healthy subjects, but the Back Mapper requires more experienced practice to have good inter-reliability. Also, the variables of the Back Mapper does not seem as appropriate compared with the Cobb angle by X-ray.

Key Words: Cobb angle, Rasterstereography, Scoliosis, Validity and reliability

\section{Introduction}

'Having correct posture' indicates balance maintenance of the musculoskeletal system. Proper alignment of the spine should be considered as a base in order to exhibit correct posture [1]. The correct posture was emphasized, because the correct alignment of the spine and pelvis exert a significant impact on the body function and musculoskeletal system. Correct alignment should be used as an objective method of evaluating the shape of the spine [2].

Reduced body movement lead to increasing transformation and disease of the spine, in accordance with the devel- opment of modern civilization and more convenient life $[3,4]$. If improper lifestyle habits persist for an extended period of time, it could impose a negative influence on the spine. Recently, scoliosis is considered a typical spine disease affecting 103,000 people in 2006 to 116,000 people in 2010 , which is a $12.2 \%$ increase over five years (Health Insurance Review \& Assessment Service of KOREA, 2011).

Scoliosis is defined by a Cobb's angle greater than 10 degrees with structural changes or otherwise one or more vertebrae deviated laterally or rotated $[2,5]$. In accordance with increasing curvature of the spine, visible problems such as height of the shoulder or pelvis being different from each

Received: 1 March, 2016 Revised: 22 March, 2016 Accepted: 22 March, 2016

Corresponding author: Dae-Sung Park

Department of Physical Therapy, Konyang University, 158 Gwanjeodong-ro, Seo-gu, Daejeon 35365, Republic of Korea Tel: 82-42-600-6419 Fax: 82-42-600-6420 E-mail: daeric@naver.com

(c) This is an Open-Access article distributed under the terms of the Creative Commons Attribution Non-Commercial License (http://creativecommons.org/licens es/by-nc/4.0) which permits unrestricted non-commercial use, distribution, and reproduction in any medium, provided the original work is properly cited.

Copyright $\odot 2016$ Korean Academy of Physical Therapy Rehabilitation Science 
other and trunk tilting may lead to difficulty participating in social activities. Also, increased curvature of the spine could impose a problem with cardiopulmonary function.

The Spinal Mouse [6] and attached landmark in the trunk and scapulae [7] is a method of measuring scoliosis. The Spinal Mouse has limited ability to assess the spine in 3 dimension. Although rastersterography had been studied, which is a non-invasive spinal analysis method, radiology is still the standard method for assessment of spinal deformity. Rasterstereography is a method for analyzing the spine in 3 dimension by scanning the back surface. In previous studies, inter-rater reliability was evaluated by using the kyphotic angle, lordotic anlge, trunk length, and trunk inclination. The results were appropriate to evaluate the shape of the spine [8]. The Cobb's angle is calculated by the King's type I or II [9]. Currently, X-ray is a common method used, but frequent radiation exposure can pose a problem [10]. Also, it is difficult to assess the spine in 3 dimension by X-ray. Therefore, the Back Mapper is a device that has been introduced to address these problems.

The Back Mapper is a three-dimensional spinal diagnostic imaging system to measure the formation of the spine. It measures the shape of the spine using inference sensors by the rasterstereography method. It is a device that measures the shape and position of the spine, and pelvic torsion. However, this device has not been proven for its reliability and validity. The aim of this study was to investigate the validity and reliability of the Back Mapper and compare with not only the former clinical assessment tools but also with the Spinal Mouse that had been used earlier.

\section{Methods}

\section{Subjects}

A total of twenty healthy students were recruited from $\mathrm{K}$ University. Subjects who were unable to stand due to neurological or musculoskeletal disease, had experienced a fracture in the spine or lower extremity, or had tattoos or open wounds on the back were excluded from the study. The subjects consisted of 5 males and 15 females. The mean age was 20.80 (1.61) years, the mean height was $164.55(8.17) \mathrm{cm}$, and the mean body weight was 58.55 (8.77) $\mathrm{kg}$ (Table 1). Subjects were fully informed of the study purpose and content prior to the study.

\section{Materials}

All 20 subjects were measured by the Back Mapper
(ABW GmbH Co., Frickenhausen, Germany) and the Spinal Mouse (Idiag, Volkerswill, Switzerland) by a physical therapist and 18 subjects had an X-ray taken by a medical doctor. The subjects were diagnosed with scoliosis if they had a Cobb's angle of 10 degrees or more, three unilateral scoliosis, five bilateral scoliosis showed. For intra-rater reliability, measurements were repeated twice by examiner A within 2 days, and for inter-rater reliability, measurements were repeated twice by examiners A and B within 2 days. All examiners were familiar with the measurement method of the Back Mapper and the Spinal Mouse, and had carried out the measurements independently in each setting.

\section{Back Mapper}

Subjects were measured by exposing the upper extremity and pelvic area, and were instructed to stand with their feet shoulder-width apart in a dark room with curtains closed. Necklaces, earrings, and hairbands were eliminated to minimize reflection of the objects. With the subject's back towards the camera, measurements were performed with the subjects in a neutral upright standing posture, with arms hanging down laterally to the body, and barefooted in a comfortable standing position with knees extended. Marking the position of the feet ensured reproducibility of the foot position (Figure 1A). A metal bar had been placed between the camera and the subject's toes to maintain the same distance of $135 \mathrm{~cm}$. Examiner A had manually placed reflect markers on $\mathrm{C} 7$, both sides of the scapular upper and lower ends, bilateral posterior superior iliac spine (PSIS), the between the PSIS, and the center of the sacrum, totaling up to 9 reflect markers placed. The analysis was performed by the ABW-MED ver.3 (ABW GmbH Co.) stored in the computer. The variables assessed were trunk inclination, trunk imbalance, pelvic position, pelvic torsion, pelvic rotation, rotation of scapulae, position of shoulder left and right, thoracic angle, lumbar angle, kyphotic angle, and lordotic angle [11].

Table 1. General characteristics of subjects

$(\mathrm{N}=20)$

\begin{tabular}{lc}
\hline \multicolumn{1}{c}{ Characteristic } & Value \\
\hline Gender (male/female) & $5 / 15$ \\
Age $(\mathrm{y})$ & $20.80(1.61)$ \\
Height $(\mathrm{cm})$ & $164.55(8.17)$ \\
Weight $(\mathrm{kg})$ & $58.55(8.77)$ \\
Cobb's angle & $7.96(4.97)$ \\
\hline
\end{tabular}

Values are presented as number only or mean (SD) 


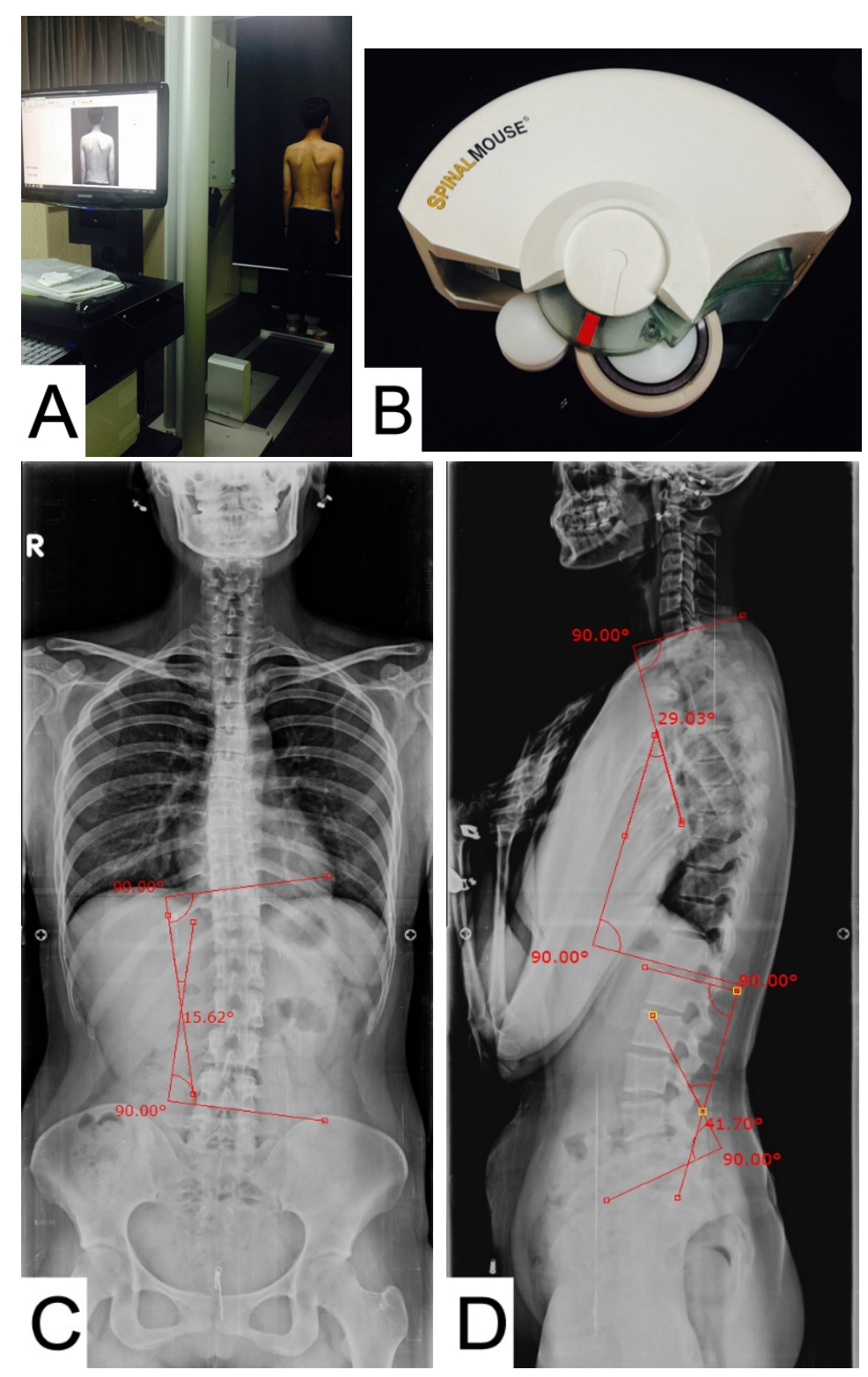

Figure 1. Back Mapper (A) Spinal Mouse (B) Cobb angle (C) kyphotic angle (D) X-ray.

\section{Spinal Mouse}

The Spinal Mouse is a non-invasive measurement equipment that measures the spine shape and angle in the frontal plane and sagittal plane (Figure 1B). Subjects stood in the anatomical position with the upper extremity exposed. Using the Spinal Mouse a perpendicular scan along the transverse process of the $\mathrm{C} 7$ to $\mathrm{L} 5$ was performed. Consequently, it is able to measure the curvature of the spine in the frontal plane. The Spinal Mouse provided the data to a computer wirelessly. Using two wheels, measurements along the spinal transverse process, distance, and angles were transmitted to a personal computer. Data was sampled every $1.3 \mathrm{~mm}$, with the number of samples set to $150 \mathrm{~Hz}$. In previous stud- ies [6], the inter-rater and intra-rater reliability of the Spinal Mouse was excellent (intraclass correlation coefficient [ICC]= 0.872-0.962). The kyphotic and lordotic angular values were collected from the measured data.

\section{X-ray}

Cobb's angle is an objective method of spinal deformity in which it is the angle formed between a line drawn parallel to the superior endplate of one vertebrae above the fracture and a line drawn parallel to the inferior endplate of the vertebrae one level below the fracture [12]. X-rays of $\mathrm{C} 1$ to the coccyx were taken in the frontal plane. The cranial and caudal vertebrae were marked in the X-ray. Afterwards, a line 
was drawn from the endplate of each spine to measure the largest angle [13]. The kyphotic angle on the X-ray was measured by drawing a line from the upper end-plate of $\mathrm{T} 1$ body and the lower end-plate. The angle of intersected lines was measured. The lordotic angle was measured by drawing a line from the upper end-plate of S1 and from the lower. The angle of the intersected lines was measured [14].

\section{Method of analysis}

The SPSS ver. 17.0 (SPSS Inc., Chicago, IL, USA) was used for method of analysis. Inter-rater reliability and intra-rater reliability was analyzed by using ICC. The validity was analyzed by correlation between the Back Mapper, Spinal Mouse and Cobb's angle. Values in the range of 0.75-1.00 indicate excellent reliability, 0.60-0.74 indicate good reliability, and less than 0.40 indicates poor reliability [15]. The standard error of measurement (SEM) tools was calculated to provide the individual prediction errors related to treatment and to assess the error of measurement tools. Our study followed the SEM $\%=\left(\mathrm{SD} \times \sqrt{1-R_{1}} \times 1 /\right.$ mean $) \times$ 100. In this formula, the SD indicates standard deviation, ICC indicates reliability. With repeated measurements, the SEM was multiplied by the square root of the number of measurement tools.

\section{Results}

\section{Reliability analysis}

The ICC for Back Mapper was high except for the pelvic position and lordotic angle of examiner A's intra-rater reli- ability measurement (Table 2). The ICC of the Back Mapper was low except for trunk imbalance, rotation of scapulae, thoracic angle, lumbar angle and kyphotic angle of examiner A with B 's inter-rater reliability measurement (Table 3 ). It showed a high reliability compared with intra-rater reliability and inter-rater reliability values for trunk imbalance, rotation of scapulae, thoracic angle, lumbar angle and kyphotic angle.

\section{Validity analysis}

The correlation between the kyphotic angle and lordotic angle in the Spinal Mouse and Back Mapper is shown in Table $4(p<0.05)$. There was a significant correlation with the kyphotic angle $(\mathrm{r}=0.51)$. The correlation between $\mathrm{X}$-ray and the Back Mapper is shown in Table 5. The Cobb's angle from the X-ray was correlated with trunk inclination on the Back Mapper $(\mathrm{r}=0.532)$. The kyphotic angle from the X-ray was correlated with the thoracic $(r=0.621)$ and lumbar angles $(r=0.693)$ on the Back Mapper.

\section{Discussion}

The aim of this study was to assess the reliability and validity and to compare the Back Mapper, Spinal Mouse and Cobb's angle to assess for scoliosis. In this study, the interrater reliability and intra-rater reliability was assessed. Regarding good inter-rater and intra-rater reliability, the Back Mapper proved to be more reliable if used by the same examiner.

A previous study [8], measured the spine using the raster-

Table 2. Intra-rater reliability of the Back Mapper between two days

$(\mathrm{N}=20)$

\begin{tabular}{|c|c|c|c|c|c|c|}
\hline \multirow{2}{*}{ Variable } & \multicolumn{2}{|c|}{ Mean (SD) } & \multirow{2}{*}{ Cronbach's $\alpha$} & \multirow{2}{*}{ ICC } & \multirow{2}{*}{ SEM } & \multirow{2}{*}{$95 \% \mathrm{CI}$} \\
\hline & A1 $(n=20)$ & A2 $(n=20)$ & & & & \\
\hline Trunk inclination $\left(^{\circ}\right)$ & $-1.65(1.57)$ & $-1.85(2.08)$ & 0.821 & 0.696 & 0.093 & $0.377-0.868$ \\
\hline Trunk imbalance $\left(^{\circ}\right)$ & $-0.05(1.23)$ & $-0.25(1.21)$ & 0.932 & 0.873 & 0.004 & $0.708-0.948$ \\
\hline Pelvic position $\left({ }^{\circ}\right)$ & $0.75(1.99)$ & $1.05(1.43)$ & 0.860 & 0.754 & 0.102 & $0.477-0.895$ \\
\hline Pelvic torsion $\left(^{\circ}\right)$ & $-0.35(2.54)$ & $-0.10(2.59)$ & 0.968 & 0.937 & 0.009 & $0.848-0.975$ \\
\hline Pelvic rotation $\left({ }^{\circ}\right)$ & $0.85(2.85)$ & $0.50(3.00)$ & 0.855 & 0.746 & 0.018 & $0.463-0.891$ \\
\hline Rotation of scapulae $\left({ }^{\circ}\right)$ & $1.45(3.25)$ & $0.90(3.46)$ & 0.899 & 0.816 & 0.038 & $0.592-0.923$ \\
\hline Position of shoulder left $\left({ }^{\circ}\right)$ & $29.87(8.40)$ & $29.39(9.51)$ & 0.965 & 0.932 & 0.203 & $0.817-0.976$ \\
\hline Position of shoulder right $\left({ }^{\circ}\right)$ & $30.95(9.41)$ & $30.55(7.48)$ & 0.955 & 0.913 & 0.353 & $0.794-0.965$ \\
\hline Thoracic angle $\left({ }^{\circ}\right)$ & $11.50(2.95)$ & $11.40(2.95)$ & 0.904 & 0.825 & 0.000 & $0.609-0.927$ \\
\hline Lumbar angle $\left(^{\circ}\right)$ & $8.95(2.47)$ & $8.85(2.80)$ & 0.956 & 0.916 & 0.060 & $0.801-0.966$ \\
\hline Kyphotic angle $\left({ }^{\circ}\right)$ & $42.90(8.20)$ & $42.90(8.19)$ & 0.984 & 0.969 & 0.002 & $0.925-0.988$ \\
\hline Lordotic angle $\left(^{\circ}\right)$ & $30.25(12.12)$ & $27.85(7.87)$ & 0.316 & 0.187 & 0.778 & $-0.267-0.574$ \\
\hline
\end{tabular}

ICC: intraclass correlation coefficient, SEM: standard error of measurement, CI: confidence interval. 
Table 3. Inter-rater reliability of the Back Mapper between examiner A and B $(\mathrm{N}=20)$

\begin{tabular}{|c|c|c|c|c|c|c|}
\hline \multirow{2}{*}{ Variable } & \multicolumn{2}{|c|}{ Mean (SD) } & \multirow{2}{*}{ Cronbach's $\alpha$} & \multirow{2}{*}{ ICC } & \multirow{2}{*}{ SEM } & \multirow{2}{*}{$95 \% \mathrm{CI}$} \\
\hline & A1 $(n=20)$ & $B(n=20)$ & & & & \\
\hline Trunk inclination $\left({ }^{\circ}\right)$ & $-1.65(1.57)$ & $-1.85(2.28)$ & 0.733 & 0.578 & 0.130 & 0.194-0.809 \\
\hline Trunk imbalance $\left({ }^{\circ}\right)$ & $-0.05(1.23)$ & $-0.25(1.02)$ & 0.870 & 0.770 & 0.038 & $0.506-0.902$ \\
\hline Pelvic position $\left({ }^{\circ}\right)$ & $0.75(1.99)$ & $0.70(1.68)$ & -0.470 & -0.023 & 0.057 & $-0.451-0.414$ \\
\hline Pelvic torsion $\left(^{\circ}\right)$ & $-0.35(2.54)$ & $-0.80(1.99)$ & 0.342 & 0.206 & 0.101 & $-0.249-0.587$ \\
\hline Pelvic rotation $\left({ }^{\circ}\right)$ & $0.85(2.85)$ & $0.40(2.85)$ & 0.648 & 0.480 & 0.000 & $0.059-0.756$ \\
\hline Rotation of scapulae $\left({ }^{\circ}\right)$ & $1.45(3.25)$ & $0.80(3.14)$ & 0.887 & 0.798 & 0.020 & $0.557-0.915$ \\
\hline Position of shoulder left $\left({ }^{\circ}\right)$ & $29.87(8.40)$ & $28.55(3.66)$ & 0.531 & 0.362 & 0.868 & $-0.146-0.719$ \\
\hline Position of shoulder right $\left({ }^{\circ}\right)$ & $30.95(9.41)$ & $29.85(4.57)$ & 0.594 & 0.422 & 0.886 & $-0.013-0.723$ \\
\hline Thoracic angle $\left({ }^{\circ}\right)$ & $11.50(2.95)$ & $11.50(2.74)$ & 0.876 & 0.779 & 0.038 & 0.523-0.906 \\
\hline Lumbar angle $\left({ }^{\circ}\right)$ & $8.95(2.47)$ & $8.90(2.57)$ & 0.935 & 0.878 & 0.027 & $0.717-0.950$ \\
\hline Kyphotic angle $\left({ }^{\circ}\right)$ & $42.90(8.20)$ & $42.55(7.84)$ & 0.958 & 0.919 & 0.066 & 0.807-0.967 \\
\hline Lordotic angle $\left(^{\circ}\right)$ & $30.25(12.12)$ & $29.40(7.47)$ & 0.527 & 0.357 & 0.851 & $-0.089-0.684$ \\
\hline
\end{tabular}

ICC: intraclass correlation coefficient, SEM: standard error of measurement, CI: confidence interval.

Table 4. The correlation in kyphotic angle and lordotic angle between Back Mapper and Spinal Mouse

$(\mathrm{N}=20)$

\begin{tabular}{ccc}
\hline \multirow{2}{*}{ Spinal Mouse } & \multicolumn{2}{c}{ Back Mapper } \\
\cline { 2 - 3 } & Kyphotic angle & Lordotic angle \\
\hline $\begin{array}{l}\text { Kyphotic angle }\left(^{\circ}\right) \\
\text { Lordotic angle }\left({ }^{\circ}\right)\end{array}$ & $0.510^{*}$ & -0.351 \\
\hline${ }^{*} p<0.05$. & &
\end{tabular}

stereographic system with and without reflective markers in 50 healthy subjects who were divided into two groups. The intra-day Cronbach's $\alpha$ was $0.742-0.987$ and the ICC was 0.734-0.971 except for torso rotation. The inter-day Cronbach's $\alpha$ was $0.739-0.983$ except for pelvis rotation, and the ICC was $0.700-0.963$ except for left deviation and the pelvis rotation. The ICC for pelvic position for intra-rater reliability of the Back Mapper was 0.586 and the ICC of the lordotic angle was 0.187 , which was considered to have low reliability. There were high ICC values except for the pelvic position and lordotic angle.

In this study, the inter-rater reliability test indicated a low reliability with a Cronbach's $\alpha$ value of -0.470 for the pelvic position, 0.342 for pelvic torsion, 0.531 for left shoulder, and 0.594 for right shoulder position. The ICC values were high for trunk imbalance, which was $0.870,0.887$ for rotation of scapulae, 0.876 for thoracic angle, 0.935 for lumbar angle, and 0.958 for kyphotic angle. Based on the results, the Back Mapper demonstrated to have high intra-rater reliability values as similarly shown from previous studies.
Table 5. The correlation of X-ray radiography and Back Mapper $(\mathrm{N}=20)$

\begin{tabular}{lcccc}
\hline \multirow{2}{*}{ X-ray } & \multicolumn{4}{c}{ Back Mapper } \\
\cline { 2 - 5 } & $\begin{array}{c}\text { Trunk } \\
\text { inclination }\end{array}$ & $\begin{array}{c}\text { Thoracic } \\
\text { angle }\end{array}$ & $\begin{array}{c}\text { Lumbar } \\
\text { angle }\end{array}$ & $\begin{array}{c}\text { Lordotic } \\
\text { angle }\end{array}$ \\
\hline Cobb's angle $\left({ }^{\circ}\right)$ & $0.532^{*}$ & & & \\
Kyphotic angle $\left({ }^{\circ}\right)$ & & $0.621^{*}$ & $0.693^{*}$ & \\
Lordotic angle $\left({ }^{\circ}\right)$ & & & $0.573^{*}$ & 0.493 \\
${ }^{*} p<0.05$. & & & & \\
& & &
\end{tabular}

However, it is suspected that attaching the reflex marker is a key-point to determine the variation judging that get a lower value in inter-rater reliability test. It is suspected that the low inter-rater reliability values were due to the attachment of the reflex markers, which could be the key point.

The Spinal Mouse is an external non-invasive measurement device that evaluates for scoliosis. A previous study on 51 subjects ages 9-18 years with adolescent idiopathic scoliosis (AIS) were measured by the Spinal Mouse while standing in the anatomical position by two different therapists who had 28 and 3 years of clinical experience. The results showed a high correlation, with an inter-rater ICC value of 0.928 , a 95\% CI of 0.873-0.959, and intra-rater ICC value of 0.944 , and a $95 \%$ CI of $0.901-0.968$. In addition, a high correlation between the Spinal Mouse and the Cobb's angle has been shown [6]. Our study has shown that there is a correlation with the Back Mapper and the Spinal Mouse. Especially, kyphotic angle of Back Mapper and kyphotic angle 
of Spinal Mouse showed significantly correlation $(\mathrm{r}=0.510)$.

The Cobb's angle is a standard method of measuring for scoliosis [16]. A previous study included subjects with juvenile idiopathic scoliosis (JIS) and AIS with a Cobb's angle of greater than 10 degrees. The results showed that the intra-rater reliability of an observer in subjects with JIS was 0.600 and 0.969 for AIS. The inter-rater reliability of an observer in subjects with JIS was 0.527 and 0.963 for AIS [12]. In our study, a correlation of 0.532 between the Cobb's angle and the Back Mapper has appeared in trunk inclination. Trunk inclination in the Back Mapper is considered to be a lateral side trunk inclination, which is an inclination from $\mathrm{C} 7$ to midpoint of bilateral PSIS on the vertical line of the lateral side. Ultimately, it can be inferred that trunk inclination is associated with the Cobb's angle [12], which measures the two severely inclined vertebrae to examine for deformation of the spine.

In this study, there was a correlation between the kyphotic and the thoracic angle $(\mathrm{r}=0.621)$, and lumbar angle $(\mathrm{r}=0.573)$ in the Back Mapper. The thoracic angle in Back Mapper is the angle formed by the vertical lines from $\mathrm{C} 7$ to the kyphotic peak of the spine of T6. The lumbar angle is the angle formed by the vertical lines from the kyphotic peak of the spine of T6 to the lordotic peak of the spine of L2. The lordotic angle is angle of the flexion point below the PSIS and valley under the kyphotic peak of the spine of T12. Accordingly, a correlation between the thoracic angle, lumbar angle and lordotic angle in the Back Mapper can be found by comparing the kyphotic angle between T1's upper end-plate and T12's lower end-plate and the lordotic angle between S1's upper end-plate and T12's lower end-plate [14].

Our study had the following limitations. First, there were a limited number of subjects. The subjects included were men and women in their twenties, which would make it difficult to apply the results to the general population. Second, this study included the twelve healthy subjects and eight subject diagnosed with scoliosis after X-ray evaluation for the study.

This study showed a high reliability inter-rater and intra-rater reliability and validity measurement for the Back Mapper, which was compared with the Spinal Mouse and Cobb's angle. There were some limitations in the diagnosis for scoliosis because the only correlation that existed between the Cobb's angle and the Back Mapper was trunk inclination. However, this is determined to be useful for comparison of other tools to diagnose for scoliosis and for evaluating the therapeutic effects before and after the intervention.

Conclusions are as follows in this study of reliability and validity by comparing Back Mapper and Spinal Mouse, Cobb's angle. Inter-rater reliability of the Back Mapper showed a high value, inter-rater reliability of the Back Mapper was showed relatively low value. As a result of the comparison with Back Mapper and Spinal Mouse, Cobb's angle, X-ray showed a significant correlation was found. On these results, Back Mapper is hard to diagnose, but it's meaningful to observing the intervention progress after diagnose the scoliosis.

These findings provide good intra-reliability of Back Mapper in healthy subject, but Back Mapper require more experienced practice and careful examination of patients. Also, the variables of Back Mapper does not seem appropriate comparison with Cobb's angle in X-ray.

\section{Conflict of Interest}

The authors declared no potential conflicts of interest with respect to the authorship and/or publication of this article.

\section{References}

1. Park GD. The effect of pelvic correction and swiss-ball exercise on pelvic tilt and pelvic torsion in the pelvis deformation of female univ. students. Korean J Phys Edu 2007;46:573-80.

2. Bunnell WP. Spinal deformity. Pediatr Clin North Am 1986;33: 1475-87.

3. Winter RB. Adolescent idiopathic scoliosis. N Engl J Med 1986; 314:1379-80.

4. Kim YJ, Lenke LG, Bridwell KH, Kim J, Cho SK, Cheh G, et al. Proximal junctional kyphosis in adolescent idiopathic scoliosis after 3 different types of posterior segmental spinal instrumentation and fusions: incidence and risk factor analysis of 410 cases. Spine (Phila Pa 1976) 2007;32:2731-8.

5. Bradford DS. Surgical treatment of low back pain in spine instability. Chir Organi Mov 1994;79:63-8.

6. Livanelioglu A, Kaya F, Nabiyev V, Demirkiran G, Firat T. The validity and reliability of "Spinal Mouse" assessment of spinal curvatures in the frontal plane in pediatric adolescent idiopathic thoraco-lumbar curves. Eur Spine J 2016;25:476-82.

7. Odom CJ, Taylor AB, Hurd CE, Denegar CR. Measurement of scapular asymetry and assessment of shoulder dysfunction using the Lateral Scapular Slide Test: a reliability and validity study. Phys Ther 2001;81:799-809.

8. Guidetti L, Bonavolontà V, Tito A, Reis VM, Gallotta MC, Baldari C. Intra- and interday reliability of spine rasterstereography. Biomed Res Int 2013;2013:745480.

9. Richards BS, Sucato DJ, Konigsberg DE, Ouellet JA. Compar- 
ison of reliability between the Lenke and King classification systems for adolescent idiopathic scoliosis using radiographs that were not premeasured. Spine (Phila Pa 1976) 2003;28:1148-56; discussion 1156-7.

10. Dutkowsky JP, Shearer D, Schepps B, Orton C, Scola F. Radiation exposure to patients receiving routine scoliosis radiography measured at depth in an anthropomorphic phantom. J Pediatr Orthop 1990;10:532-4.

11. Schroeder J, Schaar H, Mattes K. Spinal alignment in low back pain patients and age-related side effects: a multivariate crosssectional analysis of video rasterstereography back shape reconstruction data. Eur Spine J 2013;22:1979-85.

12. Modi HN, Chen T, Suh SW, Mehta S, Srinivasalu S, Yang JH, et al. Observer reliability between juvenile and adolescent idiopathic scoliosis in measurement of stable Cobb's angle. Eur
Spine J 2009;18:52-8.

13. Gstoettner M, Sekyra K, Walochnik N, Winter P, Wachter R, Bach CM. Inter- and intraobserver reliability assessment of the Cobb angle: manual versus digital measurement tools. Eur Spine J 2007; 16:1587-92.

14. Harrison DE, Cailliet R, Harrison DD, Janik TJ, Holland B. Reliability of centroid, Cobb, and Harrison posterior tangent methods: which to choose for analysis of thoracic kyphosis. Spine (Phila Pa 1976) 2001;26:E227-34.

15. Lachin JM. The role of measurement reliability in clinical trials. Clin Trials 2004;1:553-66.

16. Sobush DC, Simoneau GG, Dietz KE, Levene JA, Grossman RE, Smith WB. The lennie test for measuring scapular position in healthy young adult females: a reliability and validity study. J Orthop Sports Phys Ther 1996;23:39-50. 